\title{
Rat liver regeneration following ablation with irreversible electroporation.
}

Alexander Golberg, Bote Bruinsma, Maria Jaramillo, Martin L. Yarmush, Basak Uygun

During the past decade, irreversible electroporation ablation (IRE) has emerged as a promising tool for the treatment of multiple diseases including hepatic cancer. However, the mechanisms behind the tissue regeneration following IRE ablation have not been investigated. Our results indicate that IRE treatment immediately kills the cells at the treatment site preserving the extracellular architecture, in effect causing in vivo decellularization. Over the course of 4 weeks, progenitor cell differentiation, through YAP and notch pathways, together with hepatocyte expansion led to almost complete regeneration of the ablated liver leading to the formation of hepatocyte like cells at the ablated zone. We did not observe significant scarring or tumor formation at the regenerated areas 6 months post IRE. Our study suggests a new model to study the regeneration of liver when the naïve extracellular matrix is decellularized in vivo with completely preserved extracellular architecture. 
1 Rat liver regeneration following ablation with irreversible

2 electroporation.

3 Alexander Golberg ${ }^{1,2,{ }^{*}}$, Bote G. Bruinsma ${ }^{1,3}$, Maria Jaramillo ${ }^{1}$, Martin L. Yarmush ${ }^{1,4}$, Basak E.

4 Uygun $^{1^{*}}$.

5

61 Center for Engineering in Medicine, Massachusetts General Hospital, Harvard Medical

7 School, and the Shriners Hospitals for Children in Boston, Boston, MA 02114

$8 \quad 2$ Porter School of Environmental Studies, Tel Aviv University, Israel, 6997801

$9{ }^{3}$ Department of Surgery (Surgical Laboratory), Academic Medical Center, University of

10 Amsterdam, Amsterdam, the Netherlands

$11{ }^{4}$ Department of Biomedical Engineering, Rutgers University, Piscataway, NJ 08854

$14{ }^{*}$ Corresponding authors:

15 Email: agolberg@tauex.tau.ac.il and buygun@mgh.harvard.edu 


\section{Abstract}

19 During the past decade, irreversible electroporation ablation (IRE) has emerged as a promising

20 tool for the treatment of multiple diseases including hepatic cancer. However, the mechanisms

21 behind the tissue regeneration following IRE ablation have not been investigated. Our results

22 indicate that IRE treatment immediately kills the cells at the treatment site preserving the extracellular architecture, in effect causing in vivo decellularization. Over the course of 4 weeks, progenitor cell differentiation, through YAP and notch pathways, together with hepatocyte expansion led to almost complete regeneration of the ablated liver leading to the formation of hepatocyte like cells at the ablated zone. We did not observe significant scarring or tumor formation at the regenerated areas 6 months post IRE. Our study suggests a new model to study the regeneration of liver when the naïve extracellular matrix is decellularized in vivo with completely preserved extracellular architecture. 
33

34

35

36

37

\section{Introduction}

Irreversible electroporation (IRE) was proposed for non-thermal tissue ablation a decade ago (Davalos, Mir \& Rubinsky, 2005). It is now an emerging tool in the interventional radiology for solid tumors ablation and has other potential medical uses in hemostasis and the treatment of wounds (Golberg \& Yarmush, 2013; Golberg et al., 2013, 2014, 2015a; Yarmush et al., 2014). Electroporation leads to an increase in cell membrane permeability following exposure to highvoltage, pulsed electric fields (Weaver \& Chizmadzhev, 1996). In IRE, the change of permeability is irreversible and results in cell death (Miller, Leor \& Rubinsky, 2005; Golberg \& Yarmush, 2013). The distinguishing characteristic of IRE from other tissue ablation methods is that it only affects the cell membrane, preserving the structure of the extracellular matrix (ECM) which results in more rapid restoration of tissue perfusion(Golberg et al., 2013). However, the cellular mechanisms of IRE-induced death remains unclear: in vivo ablation is currently thought to result from a combination of mechanisms including necrosis, caspase-dependent (apoptotic) and caspase-independent cell death (Song et al., 2014; Beebe, 2015). What is even less known, is the response of tissues to the pulsed electric fields treatment. In prior reports, we showed IRE-ablated rat skin regenerates without scars (Golberg et al., 2013, 2015a). An interesting clinical observation after IRE treatment of liver tumors is that the liver tissue assumes its normal structure much more quickly than following thermal ablation, as assessed by imaging methods. It is speculated that the reduced inflammation and fibrosis after the IRE treatment may be the underlying cause (Narayanan, 2011). Intrigued with this clinical observation, we set out to investigate liver histologically following IRE ablation to determine the cellular events constituting the tissue response.

The liver has tremendous restorative capacity and is able to regenerate after removal of $90 \%$ of organ mass (Michalopoulos, 2010) and different regenerative mechanisms have been identified in various injurious conditions. Liver regeneration after hepatectomy involves expansion of 
59

remaining hepatocytes and other liver cells to restore the initial organ mass. In the case of chronic liver injury, resident hepatic progenitor cells become active and start differentiating to replace the injured hepatocytes. The resident progenitors, sometimes called "oval cells" or "small hepatocytes", emerge from the bile ducts or canals of Hering (Oertel \& Shafritz, 2008; Turner et al., 2011; Best et al., 2015) and may differentiate into both hepatocytes and biliary cells (Español-Suñer et al., 2012; Huch et al., 2013). However, the complex signaling pathways (Taub, 2004; Fausto, Campbell \& Riehle, 2006; Michalopoulos, 2007; Böhm et al., 2010) that regulate the fate of differentiating cells in the regenerating liver are not completely clear and the relations between "oval cells", bile ducts cells and hepatocytes are debated in the literature (Greenbaum, 2011; Michalopoulos, 2012).

Here we show that liver regeneration following IRE ablation occurs through progenitor cell activation as well as hepatocyte expansion. Different from the currently used models for liver regeneration studies such as partial hepatectomy and chemical-induced liver injury, IRE kills the cells by damaging the cell membrane, preserving the ECM and tissue perfusion. We show that the preserved ECM serves as a substrate for the development of mature liver tissue. In addition, our findings suggest the use of IRE ablation as a new model to study liver regeneration. 


\section{Materials and Methods}

\section{Animal subjects}

Female Lewis rats ( $N=21,180-250 \mathrm{~g}, 6$-weeks old) were obtained from Charles River

Laboratories (Wilmington, MA). The animals were housed in individual cages with access to food and water ad libitum, and were maintained on a 12-hour light/dark cycle in a temperaturecontrolled room. All animal procedures were described in protocol number 2013N000127 and were approved by the Institutional Animal Care and Use Committee (IACUC) of the Massachusetts General Hospital and were kept in accordance with the guidelines of the National Research Council.

\section{Rat liver irreversible electroporation}

Twenty minutes prior to induction of anesthesia, $0.05 \mathrm{mg} / \mathrm{kg}$ of buprenorphine was administered subcutaneously. Rats were anesthetized by inhalation of isoflurane ( $5 \%$ in $100 \%$ oxygen) in an Ohmeda Tech 4 tabletop anesthesia apparatus connected to a standard rodent system. The animal was placed in a supine position on a sterile surgical table. The abdomen was opened with a $25 \mathrm{~mm}$ midline incision from the xyphoid process down and the sternum and abdominal wall were retracted. Two non-thermal IRE ablations were performed on different lobes using small, $7 \mathrm{~mm}$ (diameter) BTX Tweezertrodes (Harvard apparatus, MA), which reduced the concentration of the current on electrodes tips, rapid drops in the electric fields and edge thermal effects associated with the needle electrodes. In the previous work, using plate electrodes with $1 \mathrm{~cm}^{2}$ surface area, we ablated rat liver by applying 90 unipolar, rectangular electric $70 \mu \mathrm{s}$ pulses, at $150 \mathrm{Vmm}-1$ potential difference between the electrodes, at $4 \mathrm{~Hz}$. That protocol led to $0.5^{\circ} \mathrm{C}$ increase of liver temperatures after the IRE procedure (Golberg et al., 2011). The protocol used in this study was optimized in our most recent work that investigated the electric field distribution in the rat liver (Golberg et al., 2015b).The electroporation parameters used in this study were as follows: electrode separation: $3 \mathrm{~mm}$, applied voltages: 
$104360 \mathrm{~V}$, number of pulses: 99, pulse length $50 \mu \mathrm{s}$, frequency of pulse delivery: $4 \mathrm{~Hz}$. Square

105 pulses were delivered using BTX 830 pulse generator (Harvard Apparatus Inc, Holliston MA,

106 USA). Currents were measured in vivo using PicoScope 4224 Oscilloscope with Pico Current

107 Clamp (60A AC/DC) and analyzed with Pico Scope 6 software (Pico technologies Inc., UK). The

108 abdomen was irrigated with $0.9 \%$ saline solution and the incision was closed in two layers using

109 4-0 silk sutures.

110 Histology

111 Tissue specimens were harvested immediately after IRE and 24 h, $3 \mathrm{~d}, 7 \mathrm{~d}, 14 \mathrm{~d}, 28 \mathrm{~d}$ and 6 mo

112 ( $n=3$ for each time point up to 6 monthsna and $n=6$ for a 6 months time point) following the initial

113 IRE ablation. Liver samples were fixed in $10 \%$ formalin, embedded in paraffin, and cut into $5 \mu \mathrm{m}$

114 sections. Sections were stained with hematoxylin and eosin (H\&E) and Masson's trichrome

115 stains by the Pathology Core at the Massachusetts General Hospital. Slides were evaluated by

116 three separate investigators. Color images of each entire tissue section were acquired using

117 NanoZoomer Digital Pathology System (Nanozoomer 2.0-HT slide scanner (Hamamatsu,

118 Hamamatsu City, Japan).

119 Immunohistochemistry

120 Paraffin-embedded tissue sections $(5 \mu \mathrm{m})$ on glass slides were baked at $60^{\circ} \mathrm{C}$ for 30 minutes,

121 followed by deparaffinization in xylene and rehydration in graded alcohol into water. Antigen

122 retrieval was performed by boiling the slides in $10 \mathrm{mM}$ sodium citrate buffer $\mathrm{pH} 6.0$ for 30

123 minutes. Endogenous peroxidase activity was quenched with Dual Endogenous Enzyme Block

124 (DAKO, Denmark) for 5 minutes. Tissue sections were incubated with 1:100 dilution of Ki67

125 rabbit monoclonal antibody (Abcam, Cambridge MA), 1:1000 dilution of Sox9, rabbit polyclonal

126 antibody (Millipore, Bedford MA), 1:2000 dilution of $\boldsymbol{\alpha}$-smooth muscle actin ( $\boldsymbol{\alpha}-\mathrm{SMA}$ ) rabbit

127 monoclonal antibody (Millipore, Bedford MA) in 1\% TBS/BSA at room temperature inside a

128 humidified chamber for 30 minutes. After washing, slides were incubated with rabbit polymer 
129 reagent (DAKO, Denmark) for 30 min at $\mathrm{RT}$ followed by incubation with the DAB+ reagent

130 (DAKO, Denmark) with monitoring for 5-10 minutes. After washing, counterstain was done

131 using Harris type hematoxylin. Slides were briefly dehydrated and then mounted with

132 Histomount solution (Life Technologies, Grand Island, NY). Color images of each entire tissue

133 section were acquired using NanoZoomer Digital Pathology System (Nanozoomer 2.0-HT slide

134 scanner, Hamamatsu, Hamamatsu City, Japan). The number of cells that are positive for Ki67

135 was counted using the software Image $(\mathrm{NIH}, \mathrm{MA})$.

136

137 q-RT-PCR

138 Biopsies (25-30 mg) of liver tissue were used to extract RNA using the NucleoSpin kit

139 (Macherey Nagel, Germany) according to manufacturer protocols. Absorbance at $280 \mathrm{~nm}$ and

$140260 \mathrm{~nm}$ was measured using a NanoDrop 100 to obtain RNA concentration and quality.

141 Reverse transcription was performed using an ImProm II reverse transcription kit (Promega, WI)

142 following manufacturer recommendations. qRT-PCR analysis was performed in ViiA7

143 instrument (Applied Biosystems, CA) using SybrGreen system and the primers listed in Table 1.

\section{Results}

146 Pulsed electric fields cause immediate vascular congestion and rapid decellularization in

147 the exposed area

148 Pulsed electric fields were applied on separate lobes of the liver through the two flat circular 149 electrodes (Fig.1a). Immediately after the ablation, the treated sites on the liver lobes lost the 150 normal color and were distinctly white in appearance, as expected (Jarm et al., 2010) (Fig.1c).

151 Histological observation showed the complete congestion of the treated area immediately after

152 the application of electric fields. Trapped red blood cells were observed in the whole treated 153 area (Fig.1e). The extracellular structure of liver appeared preserved after the IRE. Twenty-four 
154 hours after the ablation, the treatment site was completely ablated (Fig.1f). The large vessels

155 and ductular structures were preserved. The ablated area was congested with cell debris. Rare

156 epithelial and ductular cells survived at the area of the large blood vessels (Fig.1f, arrows).

157 These survival of cells at the area of the large blood vessels supported the previously

158 developed "electric field sinks" mechanisms, which showed that large vessels cause to the rapid

159 drop of the electric field strength at the areas parallel to the major vector of the electric field

160 because of the differenced in electrical conductivities of blood, vessel wall and parenchymal

161 tissues. (Golberg et al., 2015b).

162

Early events during liver regeneration after IRE ablation

The cells in the treatment site die post IRE treatment. Regenerating areas appear within the site as early as $3 \mathrm{~d}$ (Fig.2a). These areas are composed of a non-homogenous cell populations with elongated and migratory cells (black arrowhead) and ductular cells (white arrow) (top row). The site area became smaller and filled with cells by $7 \mathrm{~d}$ (Fig.2b). The cell population is still heterogeneous in morphology, composed of ductular cells (white arrow), and large round cells (black arrow,bottom row). Cells were found actively dividing, as detected with positive Ki67 staining, and they secreted new extracellular matrix (intensive Masson's trichrome staining). Interestingly, $7 \mathrm{~d}$ after the IRE ablation some large, hepatocyte-like cells, near the regenerated area were found positive for Ki67, indicating that hepatocyte expansion contributed to the regeneration process.

175 Starting on day 3 , the regenerative spots are filled with elongated cells that are positive for activated stellate cell marker $\alpha$-smooth muscle actin ( $\alpha$-SMA). Ductular cells positive for cholangiocyte marker, Sox9, populated the treatment site suggesting the contribution of progenitor cells to liver regeneration (Furuyama et al., 2011; Kawaguchi, 2013) (Fig 3). By day 
180

181

182

observations suggest a role for progenitor cells of ductular origins in liver regeneration after IRE ablation.

\section{Progenitor cells and hepatocytes contribute to the liver regeneration after PEF ablation}

At two weeks to one month after the ablation, cell proliferation continued near and at the treated site appearance similar to ductular reaction. The size of the regenerating area decreased, probably due to contraction induced by stellate cells (Soon \& Yee, 2008) (Fig.4). Secretion of the ECM adjacent to the large vessels continued (Fig.4a). Hepatocyte proliferation at the edge area of the treated zone was shown even one month after ablation (Fig.4b, Ki67 panel black arrow). Sox9 and a-SMA positive cells were present in all regenerative spots at two weeks and one month after the ablation (Fig 5). However, the intensity of Sox9 staining in the parenchyma was reduced one month after ablation in comparison with the two weeks' time point (Fig.5a vs b), possibly suggesting the differentiation and maturation of the newly formed hepatocyte-like cells. This suggestion is corroborated by the previous study that showed the downregulation of Sox9 expression associated with hepatogenic differentiation of human liver mesenchymal stem/progenitor cells (Paganelli et al., 2014).

To further elucidate some of the possible regeneration mechanisms, quantitative reverse transcriptase polymerase chain reaction was used to analyze the level of expression of markers specific to adult progenitor cells (Cldn7, Ros1, Cdh22, Cd133, Muc1) (Yovchev et al., 2007) (Fig.6a), factors implicated in activation of progenitor cells (Scf, Sdf1, C-kit, Fgf1, Fn14,Dlk1, Tgfa, Ifny) (Fig.6b), and markers involved in notch pathway activation (Notch2, Jag1, Hes1, Sox9) (Fig.6c). We observed upregulation of all progenitor specific markers ranging from 5-fold to 2000 -fold for Muc-1 and Cdh22 respectively. Similarly, most progenitor cell activation factors except for alpha-fetoprotein (Afp) (consistent with immunohistochemistry results) and Sdf-1 were found to be upregulated with expression ranging from 1.5 to 250 -fold with respect to 
206

207

208

209

210

211

212

213

214

215

216

217

218

219

220

221

222

223

224

225

226

227

228

229

230

control samples. Finally, all genes related to YAP/Notch pathway activity were also found to be upregulated with average expression ranging from 1.5 to 75 for Hes1 and connective tissue growth factor (Ctgf), respectively. It is important to note that due to the very small sample size $(n=3)$ and high variability between samples, t-test for significance only showed $p<0.05$ for Cd133, Hes1 and Tgfa (compared to control samples), however all the markers showed upregulation with respect to control for all the samples. Because some of these factors can be expressed in a number of cells, including stellate cells and hepatocytes, their expression alone is not indicative of oval cell activation, but together with the oval marker expression data, it is highly suggestive of oval cell activation. In addition, upregulation of YAP/notch related genes suggest the possibility of progenitor cell activation through these signaling pathways.

\section{Long-term regeneration of liver lobes following PEF ablation}

Six months after the ablation, the IRE-ablated area of the liver appears macroscopically contracted (Fig. 7a). The histology showed that the ablated area regenerated, with a thin formation of fibrotic tissue (2-3 cells thick) in the treated area. Hepatocyte-like cells were present in all areas and liver structural components (vessels and ducts) were present (Fig. 7b). Importantly no tumors were observed 6 months after the ablation, suggesting that IRE ablation did not lead to uncontrolled cell division.

\section{Discussion}

Irreversible electroporation (IRE) is an emerging minimally-invasive procedure for ablation of solid tumors, where tissues are ablated by high voltage pulsed electric fields. Current clinical trials involve primarily liver and pancreatic tumors (Philips, Hays \& Martin, 2013). An interesting clinical finding suggests that IRE ablated tumors respond with significantly reduced fibrosis in comparison with standard radiofrequency ablation (Narayanan, 2011). We have previously 
231 shown that IRE ablation leads to scarless skin regeneration (Golberg et al., 2013). A unique

232 effect of IRE is that it destroys the cell membranes, but preserves the extracellular milieu

233 including extracellular matrix and microvascular structure (Golberg et al., 2015a) and in effect

234 results in in vivo decellularization of the ablated area (Phillips et al., 2012). In this in vivo study

235 in rats we found that IRE ablated zone in the liver regenerates without any scar formation as a

236 result of both progenitor cell proliferation and differentiation at the center of ablated zone and by

237 hepatocyte proliferation at the edges of the ablated zone, which are clearly demarcated.

238 The presence of a progenitor cell population within the liver has long been a topic of debate, but

239 it is widely believed that these cells, termed oval cells, exist and repair injured livers when

240 hepatocyte proliferation is impaired. Studies by (Yovchev et al., 2007) identified six markers in

241 rat livers that were unique to adult progenitor cells, all of which were found to be upregulated in

242 IRE-treated tissues 2 weeks post-ablation. To further confirm activation of oval cells we looked

243 at the expression of factors implicated in oval cell activation pathways, most of which were also

244 shown to be upregulated. Scf, Fgf1, Tgfa, Dlk1, Fn14 expressions are upregulated in liver injury

245 induced by 2-acetaminofluorene and partial hepatectomy (AAF/PH), but not in that induced by

246 partial hepatectomy alone, suggesting a role in progenitor dependent liver regeneration. Scf

247 binds to C-kit whose expression has been shown to be restricted to oval cells (Fujio et al.,

248 1994). Fgf1 expression is observed in oval cells, but also in basophilic hepatocytes, and hepatic

249 stellate cells (Lowes et al., 2003). Similarly, Tgfa expression has also been observed in stellate

250 cells after AAF/PH (Evarts et al., 1993). Dlk1 expression is observed in a subpopulation of oval

251 cells found to reside distal to the periportal region and present morphology of small hepatocytes

252 (Tanimizu et al., 2004). Fn14 expression has been observed in a few periductal cells of the adult

253 liver where hepatic cells are suggested to reside, and binds to TNF-related weak inducer of

254 apoptosis (TWEAK). It has been suggested that this interaction promotes oval cell proliferation,

255 while no mitogenic effect from TWEAK is observed in hepatocytes (lacking Fn14 expression)

256 (Jakubowski et al., 2005). Finally, while expression of Ifny is restricted to immune cells, it 
257 activates oval cell proliferation through activation of Jak/Stat pathway (Akhurst et al., 2005).

258 Increased expression of these markers in our IRE treated samples (compared to control)

259 suggests regeneration by oval cell activation.

260

261

An emerging theory suggests that a hepatocyte proliferation independent mechanism of liver

262 regeneration is through dedifferentiation of hepatocytes to a progenitor stage. This occurs

263

264

265

266

267

268

269

270

271

272

273

274

275

276

277

278

279

280

281

282 through inactivation of the Hippo pathway. Because Hippo pathway activity inhibits Yap, inactivation of Hippo results in Yap activation, and this was found to dedifferentiate adult hepatocytes, which differentiate again into hepatocytes upon reintroduction of Hippo. Because regulation of Yap is at the post-translational level, we studied activation of Yap through mRNA expression of Ctgf, as it is its most highly characterized target gene. In addition, it has been demonstrated that Yap activity leads to Notch pathway upregulation and this activity mediates hepatocyte dedifferentiation (Yimlamai et al., 2014). For this reason, we studied expression of Notch and its target genes and found that these genes are unregulated in IRE treated livers compared to normal livers. The chronicle of events that follow rat liver ablation by IRE is summarized in Figure 8.

Chronic liver disease causes approximately 30,000 deaths per year in the US alone (Habka et al., 2015). The only definitive treatment for end stage liver disease is orthotopic liver transplantation and it is limited by the severe shortage of donor organs (Habka et al., 2015). In chronic liver disease, liver cannot regenerate as usually seen in healthy livers because the hepatocyte proliferation is impaired and the hepatic progenitor cell proliferation and differentiation is not sufficient to achieve full recovery (Best et al., 2013). Previous studies achieved liver decellularization in vivo with IRE and enhanced perfusion (Sano et al., 2010). Here we show that ablation of liver in vivo with IRE leads to 1) the activation and differentiation of the progenitor cells into hepatocyte like cells and 2) division of normal hepatocytes without 
283 formation of a tumor in the long term. These studies suggest a new approach to create a

284 microenvironment inducive for progenitor-based liver regeneration and possibly functional liver

285 recovery which would be a major advance in liver regenerative medicine.

287 Our results have additional implications in the clinical use of IRE in tumor ablation. In a recent

288 review of a multi-institutional prospectively-collected registry of 150 patients undergoing 169

289 IRE ablations from 2009 through 2012, 31\% of the patients had recurrence in the median

290 follow up of 18 months. Of the total $31 \%, 10.7 \%$ were local recurrences at the ablated site

291 (Philips, Hays \& Martin, 2013). In the previous work we have shown these recurrences could be

292 driven by electric field sinks, resulting from the heterogeneous structure and conductivity of the

293 liver (Golberg et al., 2015b). The results from this work indicate that the IRE treated extracellular

294 matrix provides an environment for the activation and differentiation of progenitor cells. The role

295 the tumor derived extracellular matrix on the cell differentiation is not completely understood,

296 while some studies suggested the abnormal ECM promotes the formation of tumor

297 microenvironment, tumor angiogenesis, lymphangiogenesis, inflammation and mostly

298 interesting in the frame of this study- directly promoting cellular transformation and metastasis

299 (Lu, Weaver \& Werb, 2012). The role of the IRE spared tumor matrix in the follow up

300 recurrences is an open question and requires further research. However, it is important to point

301 out that our findings are valid only for the tested experimental conditions, which can be different

302 in the clinical environment, where partially thermal effects of IRE have been observed (Jarm et

303 al., 2010; Kos et al., 2015). In the case of a thermal effects, the regenerative pathways activated

304 could be different.

305

306 This work is the first systematic long-term study of the liver response for the IRE ablation.

307 Future work will focus on the lineage tracing of cells that contribute to regeneration. The source

308 of cells that migrate to the IRE ablated area and then differentiate to hepatocyte like cells is 
309 currently unknown. Future work in this direction will focus on labeling liver cells, bone marrow

310 cells and blood cells. Additional approaches could include transplantation of IRE decellularized

311 liver from the female to the male rat with consequent chromosome staining. Additional studies

312 will focus on the identification of the function of the finally differentiated hepatocyte like cells.

313 Although by morphology the newly formed cells at the IRE ablated area resemble mature

314 hepatocytes, their function is still to be determined.

\section{Conclusions}

317 We have shown that the ablation of a normal liver by irreversible electroporation preserves the 318 extracellular matrix, liver architecture and triggers the regenerative processes. Both progenitor 319 cells and hepatocyte mitotic pathways were found to be active in the regeneration. Progenitor 320 cells contribute to the liver regeneration at the center of the ablated zone. Hepatocyte mitosis is 321 more dominant at the edges between the normal areas of the liver and the IRE ablated zone.

322 Six months after the ablation no significant scars or tumors have been observed, suggesting 323 complete regeneration of the ablated area. The function of the newly formed hepatocyte-like 324 cells is currently unknown and should be investigated in the future studies. 
326

327

328

329

330

331

332

333

334

335

336

337

338

339

340

341

342

343

344

345

346

347

348

349

350

351

352

353

354

355

356

357

358

\section{References}

Akhurst B., Matthews V., Husk K., Smyth MJ., Abraham LJ., Yeoh GC. 2005. Differential lymphotoxin-beta and interferon gamma signaling during mouse liver regeneration induced by chronic and acute injury. Hepatology 41:327-335.

Beebe S. 2015. Mechanisms of Nanosecond Pulsed Electric Field (NsPEF)-Induced Cell Death in Cells and Tumors. J Nanomed Res 2:00016.

Best J., Dollé L., Manka P., Coombes J., van Grunsven L a., Syn W-K. 2013. Role of liver progenitors in acute liver injury. Frontiers in physiology 4:258.

Best J., Manka P., Syn W-K., Dollé L., van Grunsven LA., Canbay A. 2015. Role of liver progenitors in liver regeneration. Hepatobiliary surgery and nutrition 4:48-58.

Böhm F., Köhler UA., Speicher T., Werner S. 2010. Regulation of liver regeneration by growth factors and cytokines. EMBO molecular medicine 2:294-305.

Davalos R V., Mir LM., Rubinsky B. 2005. Tissue ablation with irreversible electroporation. Annals of Biomedical Engineering 33:223-231.

Español-Suñer R., Carpentier R., Van Hul N., Legry V., Achouri Y., Cordi S., Jacquemin P., Lemaigre F., Leclercq IA. 2012. Liver progenitor cells yield functional hepatocytes in response to chronic liver injury in mice. Gastroenterology 143.

Evarts RP., Hu Z., Fujio K., Marsden ER., Thorgeirsson SS. 1993. Activation of hepatic stem cell compartment in the rat: role of transforming growth factor alpha, hepatocyte growth factor, and acidic fibroblast growth factor in early proliferation. Cell growth \& differentiation : the molecular biology journal of the American Association for Cancer Research 4:555-561.

Fausto N., Campbell JS., Riehle KJ. 2006. Liver regeneration. Hepatology (Baltimore, Md.) 43:S45-53.

Fujio K., Evarts RP., Hu Z., Marsden ER., Thorgeirsson SS. 1994. Expression of stem cell factor and its receptor, c-kit, during liver regeneration from putative stem cells in adult rat. Laboratory investigation; a journal of technical methods and pathology 70:511-516.

Furuyama K., Kawaguchi Y., Akiyama H., Horiguchi M., Kodama S., Kuhara T., Hosokawa S., Elbahrawy A., Soeda T., Koizumi M., Masui T., Kawaguchi M., Takaori K., Doi R., Nishi E., Kakinoki R., Deng JM., Behringer RR., Nakamura T., Uemoto S. 2011. Continuous cell supply from a Sox9-expressing progenitor zone in adult liver, exocrine pancreas and intestine. Nature genetics 43:34-41.

Golberg A., Laufer S., Rabinowitch HD., Rubinsky B. 2011. In vivo non-thermal irreversible 
electroporation impact on rat liver galvanic apparent internal resistance. Physics in medicine and biology 56:951-963.

Golberg A., Broelsch GF., Bohr S., Mihm MC., Austen WG., Albadawi H., Watkins MT., Yarmush ML. 2013. Non-thermal, pulsed electric field cell ablation: A novel tool for regenerative medicine and scarless skin regeneration. Technology 1:1-8.

Golberg A., Broelsch GF., Vecchio D., Khan S., Hamblin MR., Austen WG., Sheridan RL., Yarmush ML. 2014. Pulsed Electric Fields for Burn Wound Disinfection in a Murine Model. Journal of burn care \& research : official publication of the American Burn Association.

Golberg A., Khan S., Belov V., Quinn KP., Albadawi H., Felix Broelsch G., Watkins MT., Georgakoudi I., Papisov M., Mihm MC., Austen WG., Yarmush ML. 2015a. Skin rejuvenation with non-invasive pulsed electric fields. Scientific reports 5:10187.

Golberg A., Bruinsma BG., Uygun BE., Yarmush ML. 2015b. Tissue heterogeneity in structure and conductivity contribute to cell survival during irreversible electroporation ablation by “electric field sinks". Scientific reports 5:8485.

Golberg A., Yarmush ML. 2013. Nonthermal irreversible electroporation: Fundamentals, applications, and challenges. IEEE Transactions on Biomedical Engineering 60:707-714.

Greenbaum LE. 2011. The ductal plate: a source of progenitors and hepatocytes in the adult liver. Gastroenterology 141:1152-5.

Habka D., Mann D., Landes R., Soto-Gutierrez A. 2015. Future Economics of Liver Transplantation: A 20-Year Cost Modeling Forecast and the Prospect of Bioengineering Autologous Liver Grafts. PloS one 10:e0131764.

Huch M., Dorrell C., Boj SF., van Es JH., Li VSW., van de Wetering M., Sato T., Hamer K., Sasaki N., Finegold MJ., Haft A., Vries RG., Grompe M., Clevers H. 2013. In vitro expansion of single Lgr5+ liver stem cells induced by Wnt-driven regeneration. Nature 494:247-50.

Jakubowski A., Ambrose C., Parr M., Lincecum JM., Wang MZ., Zheng TS., Browning B., Michaelson JS., Baestcher M., Wang B., Bissell DM., Burkly LC. 2005. TWEAK induces liver progenitor cell proliferation. Journal of Clinical Investigation 115:2330-2340.

Jarm T., Cemazar M., Miklavcic D., Sersa G. 2010. Antivascular effects of electrochemotherapy: implications in treatment of bleeding metastases. Expert review of anticancer therapy 10:729-746.

Kawaguchi Y. 2013. Sox9 and programming of liver and pancreatic progenitors. Journal of Clinical Investigation 123:1881-1886. 
393

394

Kos B., Voigt P., Miklavcic D., Moche M. 2015. Careful treatment planning enables safe ablation of liver tumors adjacent to major blood vessels by percutaneous irreversible electroporation (IRE). Radiology and Oncology 49:234-41.

Lowes KN., Croager EJ., Olynyk JK., Abraham LJ., Yeoh GCT. 2003. Oval cell-mediated liver regeneration: Role of cytokines and growth factors. Journal of gastroenterology and hepatology 18:4-12.

Lu P., Weaver VM., Werb Z. 2012. The extracellular matrix: A dynamic niche in cancer progression. Journal of Cell Biology 196:395-406.

Michalopoulos GK. 2007. Liver regeneration. Journal of cellular physiology 213:286-300.

Michalopoulos GK. 2010. Liver regeneration after partial hepatectomy: critical analysis of mechanistic dilemmas. The American journal of pathology 176:2-13.

Michalopoulos GK. 2012. Phenotypic fidelity (or not?) of epithelial cells in the liver. Hepatology (Baltimore, Md.) 55:2024-7.

Miller L., Leor J., Rubinsky B. 2005. Cancer cells ablation with irreversible electroporation. Technology in cancer research \& treatment 4:699-705.

Narayanan G. 2011. Irreversible electroporation for treatment of liver cancer. Gastroenterology \& hepatology 7:313-6.

Oertel M., Shafritz DA. 2008. Stem cells, cell transplantation and liver repopulation. Biochimica et biophysica acta 1782:61-74.

Paganelli M., Nyabi O., Sid B., Evraerts J., El Malmi I., Heremans Y., Dollé L., Benton C., Calderon P-B., van Grunsven L., Heimberg H., Campard D., Sokal E., Najimi M. 2014. Downregulation of Sox9 expression associates with hepatogenic differentiation of human liver mesenchymal stem/progenitor cells. Stem cells and development 23:1377-91.

Philips P., Hays D., Martin RCG. 2013. Irreversible Electroporation Ablation (IRE) of Unresectable Soft Tissue Tumors: Learning Curve Evaluation in the First 150 Patients Treated. PLoS ONE 8:e76260.

Phillips M a., Narayan R., Padath T., Rubinsky B. 2012. Irreversible electroporation on the small intestine. British journal of cancer 106:490-5.

Sano MB., Neal RE., Garcia PA., Gerber D., Robertson J., Davalos R V. 2010. Towards the creation of decellularized organ constructs using irreversible electroporation and active mechanical perfusion. Biomedical engineering online 9:83.

Song Z-Q., Xu X-H., Pan Z-H., Yao C-G., Zhou Q-H. 2014. Mechanisms for steep pulse irreversible electroporation technology to kill human large cell lung cancer cells L9981. 
International journal of clinical and experimental medicine 7:2386-94.

427 428

Soon RK., Yee HF. 2008. Stellate Cell Contraction: Role, Regulation, and Potential Therapeutic Target. Clinics in Liver Disease 12:791-803.

Tanimizu N., Tsujimura T., Takahide K., Kodama T., Nakamura K., Miyajima A. 2004. Expression of Dlk/Pref-1 defines a subpopulation in the oval cell compartment of rat liver. Gene expression patterns : GEP 5:209-18.

Taub R. 2004. Liver regeneration: from myth to mechanism. Nature reviews. Molecular cell biology 5:836-47.

Turner R., Lozoya O., Wang Y., Cardinale V., Gaudio E., Alpini G., Mendel G., Wauthier E., Barbier C., Alvaro D., Reid LM. 2011. Human hepatic stem cell and maturational liver lineage biology. Hepatology 53:1035-1045.

Weaver JC., Chizmadzhev YA. 1996. Theory of electroporation: A review. Bioelectrochemistry and Bioenergetics 41:135-160.

Yarmush ML., Golberg A., Serša G., Kotnik T., Miklavčič D. 2014. Electroporation-Based Technologies for Medicine: Principles, Applications, and Challenges. Annual review of biomedical engineering 16:295-320.

Yimlamai D., Christodoulou C., Galli GG., Yanger K., Pepe-Mooney B., Gurung B., Shrestha K., Cahan P., Stanger BZ., Camargo FD. 2014. Hippo pathway activity influences liver cell fate. Cell 157:1324-1338.

Yovchev MI., Grozdanov PN., Joseph B., Gupta S., Dabeva MD. 2007. Novel hepatic progenitor cell surface markers in the adult rat liver. Hepatology (Baltimore, Md.) 45:139-149.

(1)

(1)




\section{$451 \quad$ Figure Legends}

452 Figure 1. Ablation procedure with irreversible electroporation. Irreversible electroporation 453 setup (a), application of the electrodes on the liver lobe (b), ablated areas as appear 454 immediately after the treatment (c). Liver histology (H\&E). Normal liver (d), ablated zone 455 immediately after the treatment (e), ablated zone 24 hours after the treatment (f) with areas of 456 rare survived ductal cells (black arrows). $\mathrm{N}=3$ for each time point.
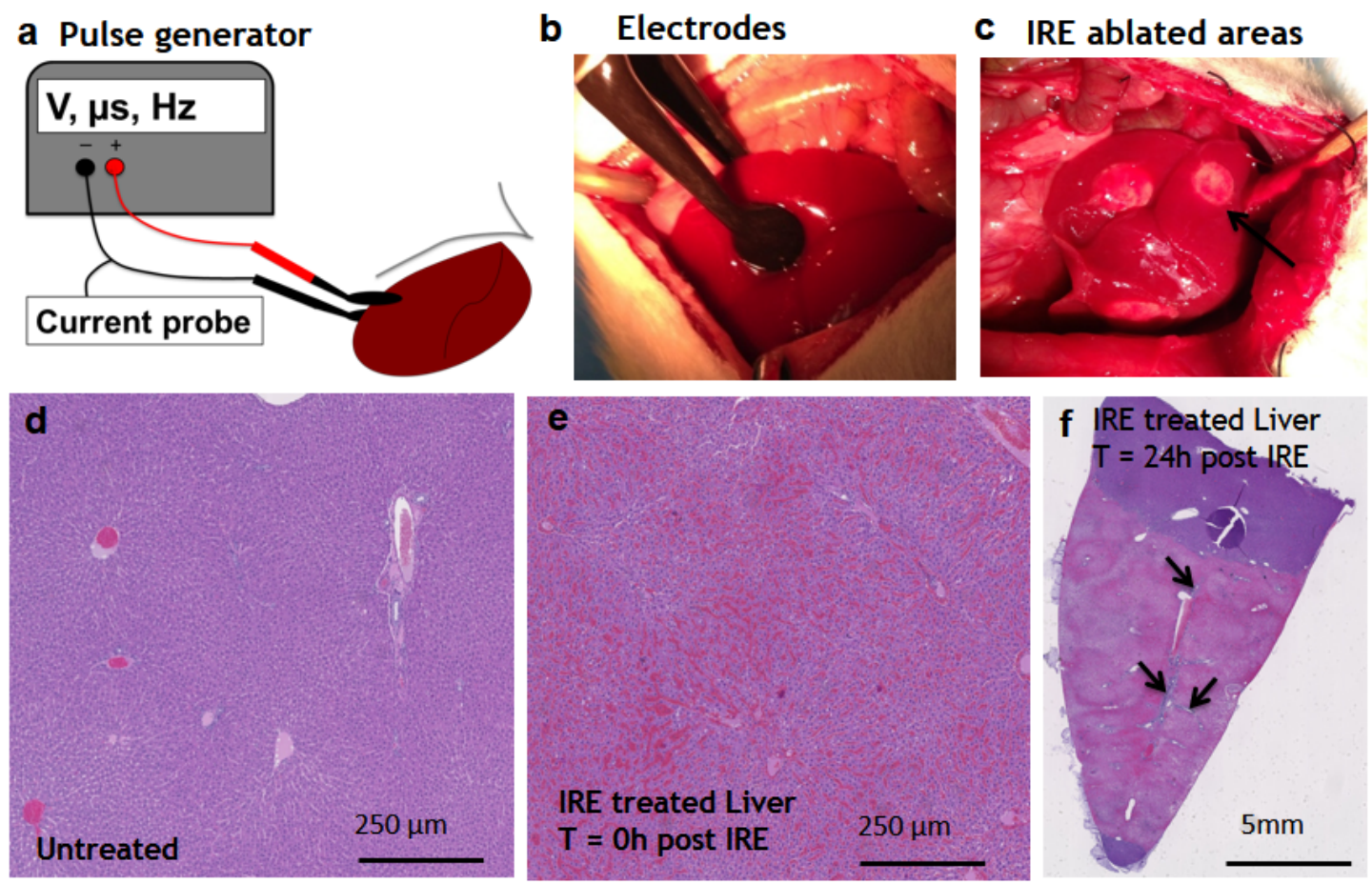
459 Figure 2. Early regeneration events following liver irreversible electroporation ablation.

460 The cells in the treatment site die post-IRE treatment. The regenerative zones appear within the

461 site as early as $3 \mathrm{~d}(\mathrm{a})$. The regenerative zones are composed of non-homogenous cell

462 populations, elongated and spindle shapes cells (black arrowhead) and ductular cells (white

463 arrow) (top row). The site is contracted and filled with cells by $7 d$ (b). The cell population is still

464 heterogeneous in morphology, composed of ductular cells (white arrow), and large cells (black

465 arrow) (bottom row). Extracellular matrix deposition is observed at the treated sites (Masson's

466 trichrome stain). Division of cells, including hepatocytes is observed as indicated with Ki67 stain.

$467 \mathrm{~N}=3$ for each time point.

468
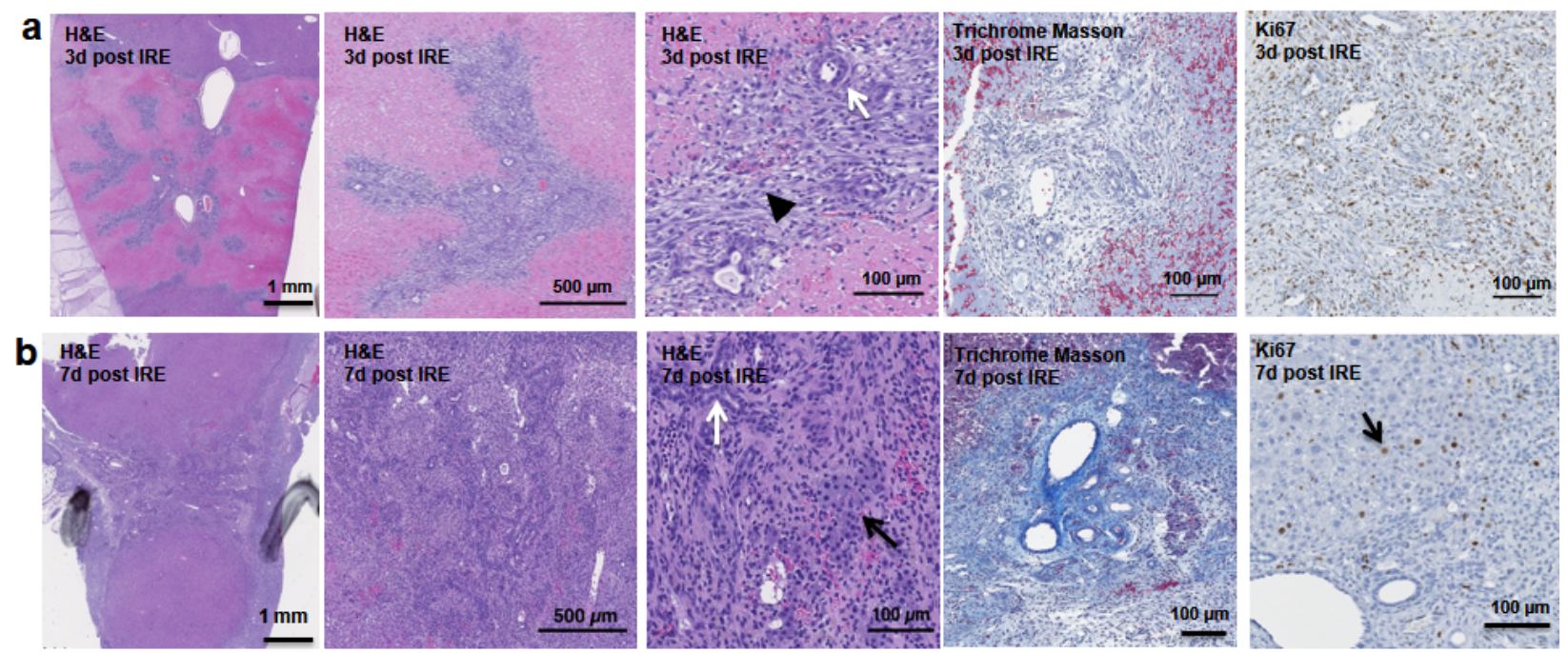
470 Figure 3. Immunohistochemistry of ablated zones- early events. On day 3 (a) and day 7 (b)

471 post ablation, Sox9 and alpha-SMA positive cells were observed. 3 days after ablation Sox9

472 positive cells were all located near the area of the ductular cells (a, left panel); however, 7 days

473 after the ablation Sox9 positive cells appear in the parenchymal tissue suggesting migration and

474 differentiation of the ductular cells to the ablated zone (b, right panel). Alpha-SMA staining was

475 associated with the non-ductular cells located near the area of vessels and ducts (a,b left

476 panel). $\mathrm{N}=3$ for each time point.

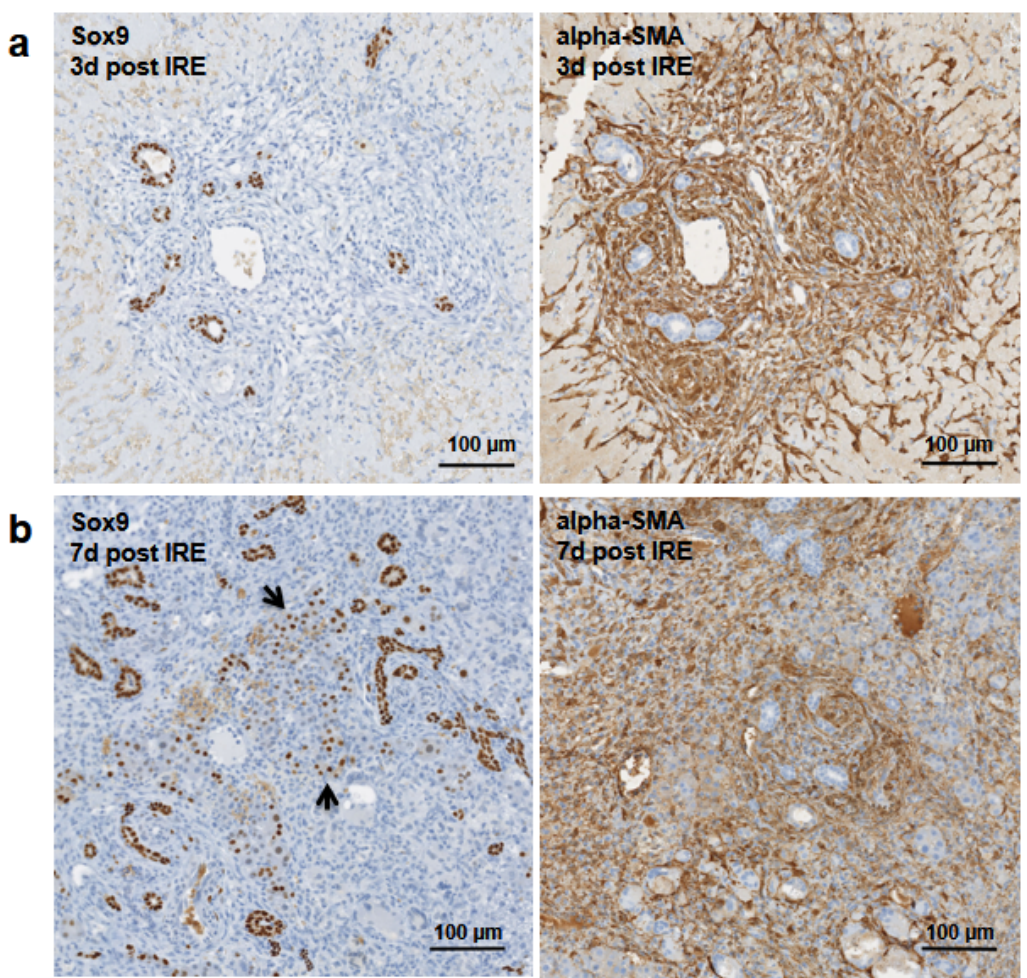


479 Figure 4. Late regeneration events following irreversible electroporation ablation. 14

480 days after the ablation, the site is still filled with ductular reactions and multiple proliferating cells

481 (a). The number of proliferating cells reduced at 28 days after the ablation (b) and disappeared

4826 months after ablation (Fig.7). Secretion of extracellular matrix, as detected by Masson's

483 trichrome staining, and active cell division, as detected by Ki67 overexpression, continued at

484 both 14 and 28 days after ablation. Ki67 staining was mostly observed at the dividing

485 hepatocyte-like steps. $\mathrm{N}=3$ for each time point.

486

a

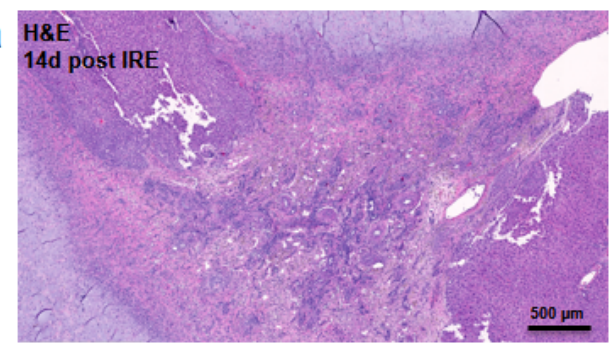

b

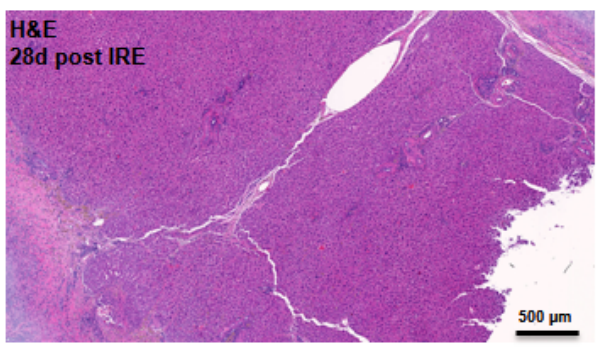

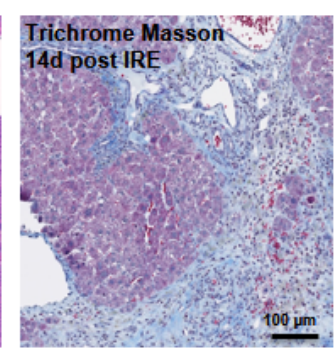
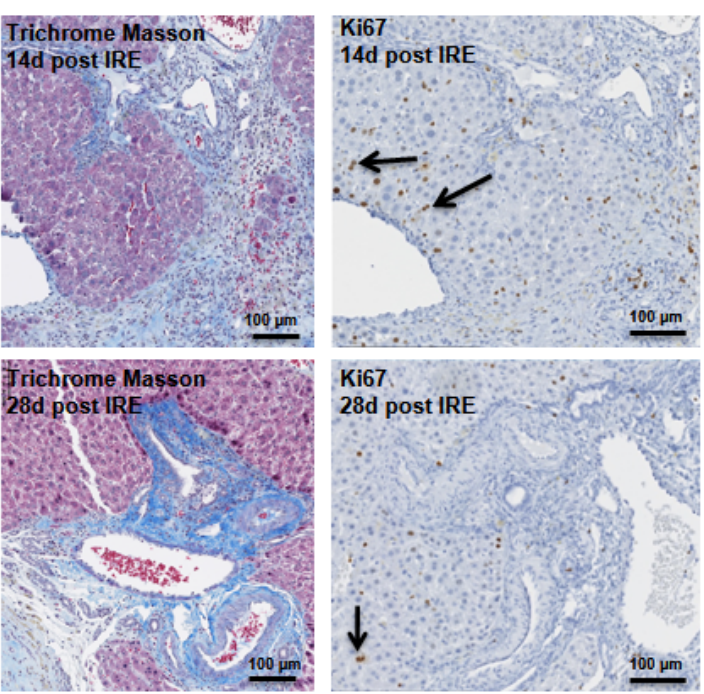
489 Figure 5. Immunohistochemistry of ablated zones- late events. 14 days after ablation (a, 490 right), multiple cells in the parenchymal tissue expressed Sox9 positive staining. However, 28 491 days (b) after ablation, the Sox9 positive staining was observed only in the ductular cells as at 492 the 3 days after ablation. Alpha-SMA staining was associated mostly with epithelial cells lining 493 the vessels at both 14 and 28 days after ablation. $\mathrm{N}=3$ for each time point.

494

a

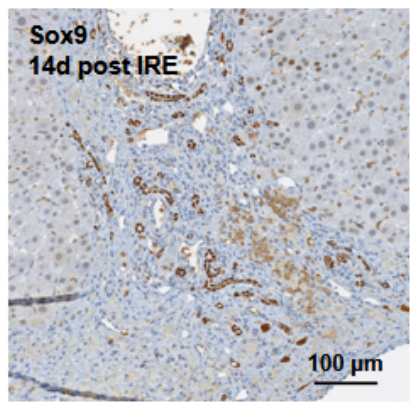

b

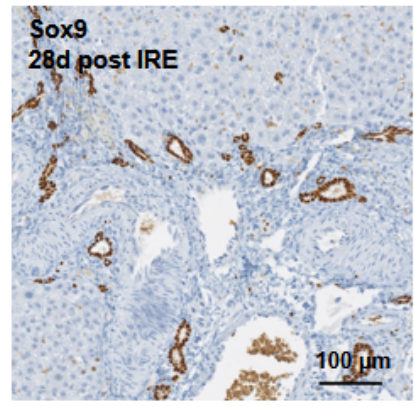

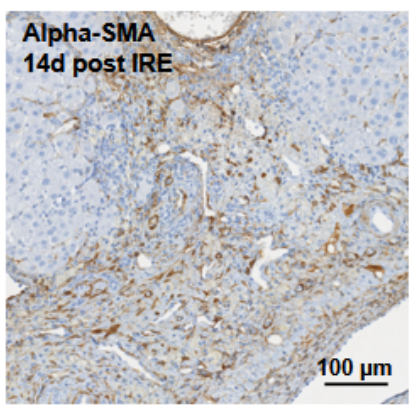

Alpha-SMA

28d post IRE

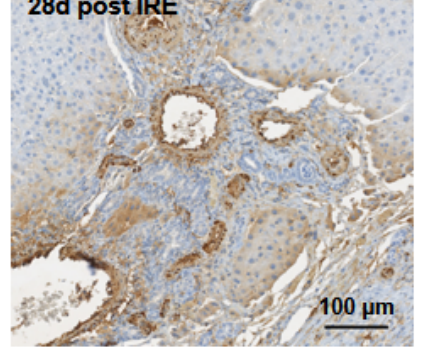


496 Figure 6. Quantitative qRT-PCT of ablated tissue 2 weeks post-IRE. 2 weeks after ablation,

497 tissue biopsies of injured site were collected and qRT-PCR to analyze expression of oval cell

498 markers and activation of pathways involved in oval cell regeneration. All oval cell specific

499 markers were found to be upregulated relative to untreated tissue (a). In addition, a large

500 number of genes related to oval cell activation were also found to be upregulated (b).

501 Upregulation of Notch2 and downstream genes was observed in IRE treated samples compared

502 to untreated controls (c). $\mathrm{N}=3$ for treatment and $\mathrm{N}=2$ for controls
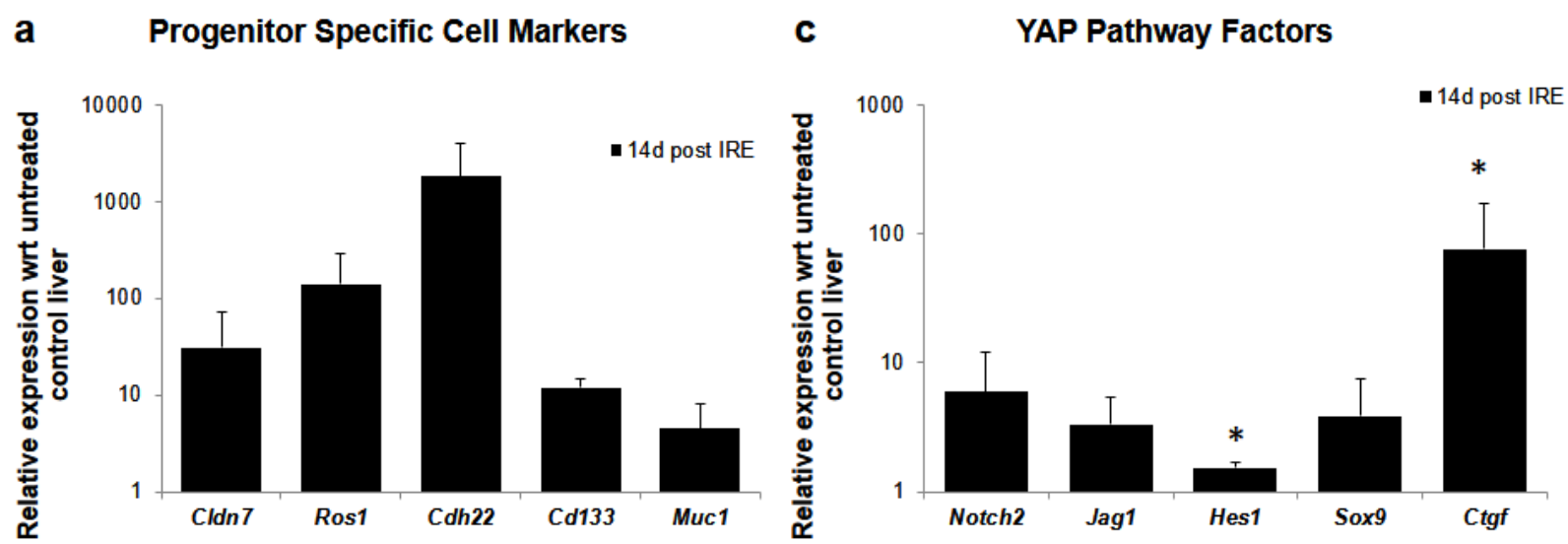

b

Progenitor Cell Activation Factors

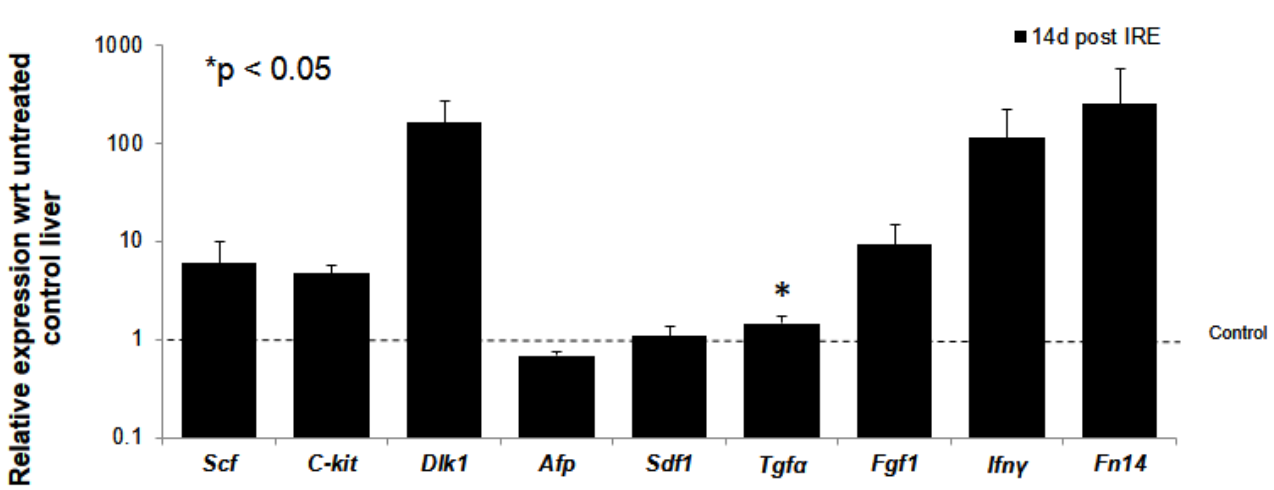


505 Figure 7. Liver regeneration 6 months after IRE ablation. Small contraction is observed at

506 the ablated site (a). The contraction is characterized by the intrusion of two-cell thick layer of

507 connective tissue inside the regenerated part of the liver (b). No tumors or fibrotic tissue is

508 observed at the regenerated zone. $\mathrm{N}=6$.

a

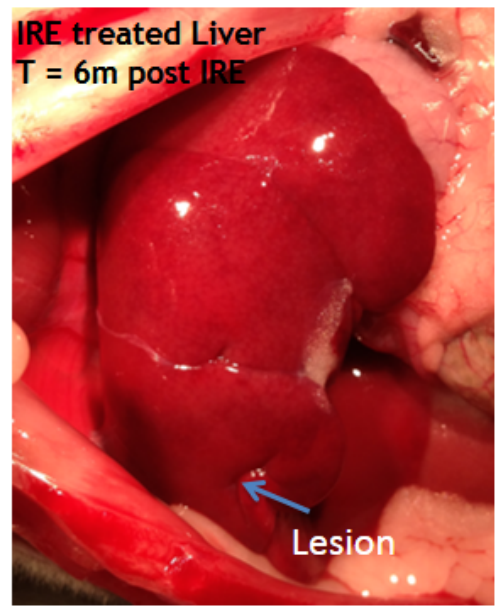

b H\&E IRE treated Liver $\mathrm{T}=6 \mathrm{~m}$ post IRE

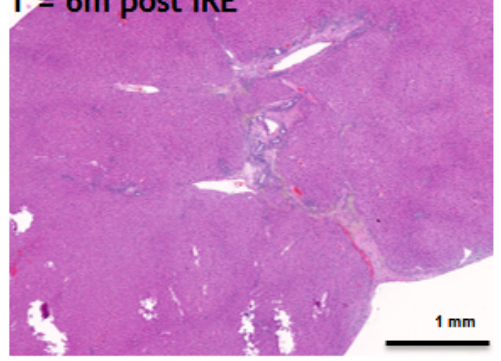


510 Figure 8. Chronicle of events following rat liver ablation by irreversible electroporation.

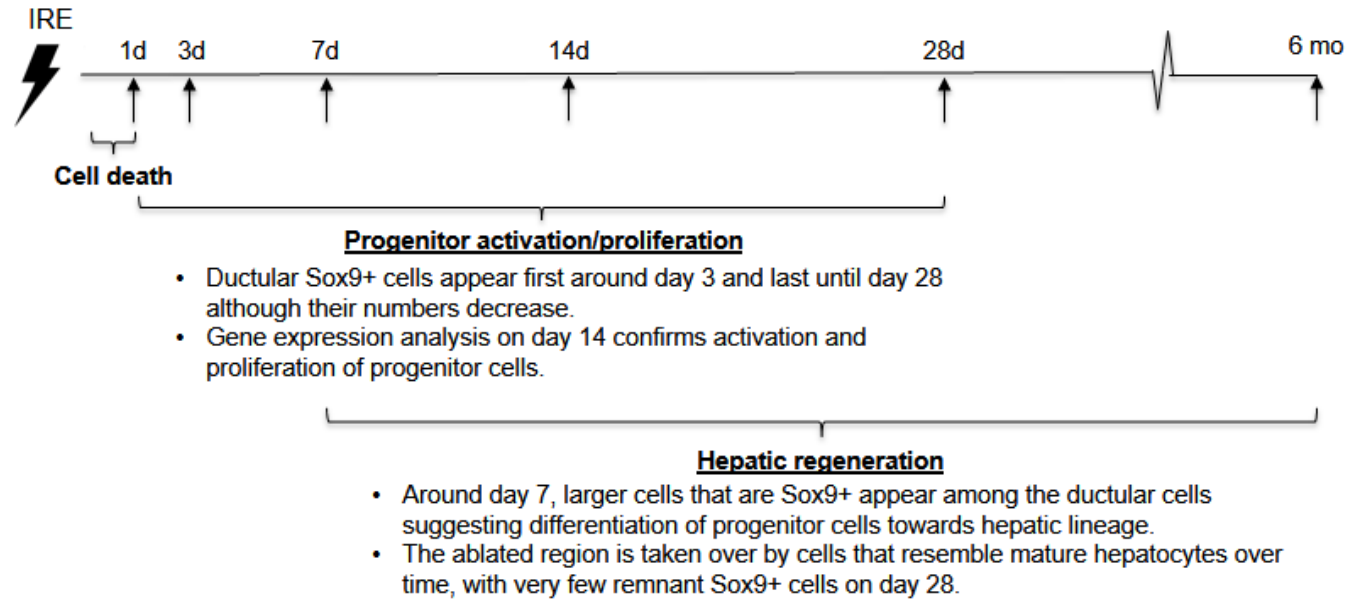

511 


\section{Table 1. Primers Used for q-RT-PCR}

\section{Primer Sequence}

Ros-1 $F$ agaaagacgagaggcaacca

Ros-1 $R$ aggagagccataagccacct

Cdh22 F catcgctctcttggtctgtg

Cdh22 R gtcataagcctcggtgtcct

Cldn7 $F \quad$ acagcccctccacttcttg

Cldn7 $R \quad$ aaccgagccaaacacaaatc

Muc1 $L \quad$ caatggcagtagcggtctct

Muc1 R gtgggtagggtgacttgctc

Cd133 F tttgtatgtgccgttgctgt

Cd133 $R \quad$ cgagtccttgtctgctggtt

Sox9 $F \quad$ agagaacgcacatcaagacg

Sox9 $R \quad$ tctggtggtcggtgtagtca

TgfaF cgcagacccaaaccataagt

TgfaFR cagggaacaacatcaccatct

Fgf1 $F \quad$ cgctggataggagatgaggt

Fgf1 $R \quad$ atggtgcgttcaagacaggt

DIk/Prefl $F$ ggaggctggtgatgaggata

DIk/Prefl $R$ ggagggaggggttcttagc

lgny $F \quad$ tcttcagcaacagtaaagcaaaa

Igny $R \quad$ cccagaatcagcaccgact

Scf $F \quad$ cctgttcttgctaccotga

Scf $R \quad$ gtgttctcgtccccatcatc

Sdf1 $F \quad$ ctttgagggagggtttggag

Sdf1 $R \quad$ tgcggaggaatgacttctgt

Fn14 $F \quad$ cagactcttccaaccacaagg

Fn14 $R \quad$ acctagcttgaggctctctgtct

Ctgf $F \quad$ gctggagaagcagagtcgtc

Ctgf $R \quad$ acaccccacagaacttagcc

Notch2 $F \quad$ cggcttcagtggtatggact

Notch $R \quad$ attcttacagggetcgetca

Jag1 $F \quad$ tctactggtgtgtgcggaag

Jag1 $R \quad$ aatccttgatggggactgtg

Hes1 $F \quad$ ggacggtgaacgactacacg

Hes1 $R \quad$ gccttcctttgtgcagag 


$\begin{array}{ll}\text { Gapdh } F & \text { ggcattgctctctcaagacaa } \\ \text { Gapdh } R & \text { atgtaggccatgaggtccac } \\ \text { C-kit } F & \text { tccctgtgaagaacactacgg } \\ \text { C-kit } R & \text { ttgctttggttgtcggattt } \\ \text { Afp } F & \text { tgttcctcattggctacacg } \\ \text { AFp } R & \text { gttcacagggtttgcctcat }\end{array}$

514

515

516 